\title{
Performance of a direct-detection active matrix flat panel dosimeter (AMFPD) for IMRT measurements
}

\author{
Yu Chen, ${ }^{\text {a) }}$ Jean M. Moran, Donald A. Roberts, Youcef El-Mohri, \\ Larry E. Antonuk, and Benedick A. Fraass \\ Department of Radiation Oncology, University of Michigan, Ann Arbor, Michigan 48109
}

(Received 10 May 2007; revised 27 September 2007; accepted for publication 17 October 2007; published 28 November 2007)

\begin{abstract}
The dosimetric performance of a direct-detection active matrix flat panel dosimeter (AMFPD) is reported for intensity modulated radiation therapy (IMRT) measurements. The AMFPD consists of $a$-Si: $\mathrm{H}$ photodiodes and thin-film transistors deposited on a glass substrate with no overlying scintillator screen or metal plate. The device is operated at 0.8 frames per second in a continuous acquisition or fluoroscopic mode. The effect of the applied bias voltage across the photodiodes on the response of the AMFPD was evaluated because this parameter affects dark signal, lag contributions, and pixel sensitivity. In addition, the AMPFD response was evaluated as a function of dose, dose rate, and energy, for static fields at $10 \mathrm{~cm}$ depth. In continuous acquisition mode, the AMFPD maintained a linear dose response $\left(r^{2}>0.99999\right)$ up to at least $1040 \mathrm{cGy}$. In order to obtain reliable integrated dose results for IMRT fields, the effects of lag on the radiation signal were minimized by operating the system at the highest frame rate and at an appropriate reverse bias voltage. Segmental MLC and dynamic MLC IMRT fields were measured with the AMFPD, and the results were compared to film, using standard methods for reliable film dosimetry. Both AMFPD and film measurements were independently converted to dose in cGy. $\gamma$ and $\chi$ values were calculated as indices of agreement. The results from the AMFPD were in excellent agreement with those from film. When $2 \%$ of $D_{\max }$ and $2 \mathrm{~mm}$ of distance to agreement were used as the criteria, $98 \%$ of the region of interest (defined as the region where dose is greater than $5 \%$ of $D_{\max }$ ) satisfied $|\chi| \leq 1$ on average across the cases that were tested. (c) 2007 American Association of Physicists in Medicine.
\end{abstract}

[DOI: $10.1118 / 1.2805993$ ]

Key words: flat panel dosimeter, amorphous silicon, dosimetry, IMRT, quality assurance

\section{INTRODUCTION}

Intensity modulated radiation therapy quality assurance (IMRT QA) is performed to ensure that the intended treatment fields obtained from the treatment planning system are correctly transferred to the treatment delivery system and accurately delivered when compared with calculations in a phantom geometry. Several methods of IMRT plan verification are used in clinical practice. ${ }^{1}$ Measurement-based verification often includes dose verification of the entire treatment plan delivery with an ionization chamber treated at the planned gantry and collimator positions plus additional twodimensional dose verification of individual IMRT fields at a standard depth, using a perpendicular gantry angle. The twodimensional measurements can be made with film or detector arrays of diodes or ionization chambers. ${ }^{2,3}$ Due to the widespread availability of amorphous silicon $(a-\mathrm{Si}: \mathrm{H})$ electronic portal imaging devices (EPIDs) for patient imaging, the use of EPIDs for IMRT QA has also been investigated. ${ }^{4-6}$

In a typical $a-\mathrm{Si}: \mathrm{H}$ flat panel imaging device with active matrix addressing using thin-film transistors (TFTs), an x-ray converter consisting of a copper plate and a phosphor scintillator is positioned on the top of the $a-\mathrm{Si}: \mathrm{H}$ circuits. The scintillator converts $\mathrm{x}$ rays and electrons emerging from the copper plate into optical photons, which are sensed by a photodiode located at every pixel. The x-ray conversion lay- ers are thick enough to produce a detectable change in the energy fluence of the incident radiation to the photodiode detectors at the pixels. Although the x-ray converter is used to improve the $\mathrm{x}$-ray detection efficiency of the imager, it has a significantly different dose response from that of waterequivalent materials, which makes the relationship between the portal dose (signal produced by the $a$-Si:H photodiodes) and the water-equivalent dose more complicated. To address this constraint, a device employing direct detection of incident radiation was developed for dosimetric measurements, ${ }^{7}$ based on an $a-\mathrm{Si}: \mathrm{H}$ panel (like that of a conventional flat panel imager), but without the x-ray converter. An initial prototype active matrix flat panel dosimeter (AMFPD) was assembled and characterized for its stability, dose response, dose rate dependence, and energy dependence for a range of situations, including IMRT field measurements in radiographic mode. ${ }^{8}$ In this operating mode, all the signal was integrated on the detector, and the entire array of pixels was read out after the radiation was completely delivered. Excellent long-term stability of dose response (less than 1.5\% variation over a year) and good dosimetric agreement between the AMFPD and film at low doses were reported. However, dark current and charge trapping led to significant artifacts in dose images for high dose dosimetry measurements when the AMFPD was operated in radiographic mode. 
In the present study, we operate the AMFPD in fluoroscopic mode, motivated by the need to understand: (1) The relationship between the dependence of dark current on charge accumulated in the photodiode and the dependence of a dose image on how the radiation is delivered (deliveryhistory dependence), and (2) the relationship between bias voltage and pixel sensitivity. In this article, the linearity of the dose response, the effect of dark current in the photodiode on the accuracy of dosimetric measurements, and the determination of two-dimensional dose distributions for IMRT fields are reported. More than 20 IMRT fields were measured, encompassing dynamic MLC (DMLC) and segmental MLC (SMLC) delivery methods, $1 \times 1 \mathrm{~cm}^{2}$ and 0.5 $\times 0.5 \mathrm{~cm}^{2}$ beamlets, and a wide range of doses for IMRT fields for prostate, head and neck, and partial breast irradiation.

\section{METHOD AND MATERIALS}

\section{II.A. The acquisition system}

The AMFPD system consists of a flat panel detector and a control computer. The detector comprised a $26 \times 26 \mathrm{~cm}^{2}$ $a$-Si: $\mathrm{H}$ array, ${ }^{9}$ data transfer electronics, and a power supply. The control computer sends instructions to the detector and stores the acquired images. The array consists of $512 \times 512$ pixels at a pixel pitch of $508 \mu \mathrm{m}$-with each pixel incorporating a photodiode that serves to sense the $\mathrm{x}$-ray signal. Each photodiode is coupled to a TFT. These TFTs act as switches that are controlled by the acquisition electronics to capture and then read out the charge stored in the photodiode-one row of pixels at a time. The gates of the TFTs along the same row are connected to a shared gate line, and the drains of the TFTs along the same column are connected to a shared data line. The photodiodes and TFTs, along with the gate and data lines, are deposited on an $\sim 1 \mathrm{~mm}$ thick glass substrate. There is no additional $\mathrm{x}$-ray converter, such as a metal plate or a scintillator, covering the surface of the photodiodes. The surface of the detector array is placed at a desired depth in a solid water phantom.

\section{II.B. Operating mode considerations}

During irradiation, the signal information extracted from an array pixel contains a dark signal component unrelated to the radiation. In order to estimate this component, dark image frames are read out in the absence of radiation. The dark signal originates from the accumulation of dark current over a frame time $T_{\text {frame }}$. The frame time is the interval between two consecutive readouts of the same row of pixels. The dark current largely corresponds to leakage current generated in the reverse-biased photodiode. It results from thermal excitation of the photodiode and depends on the magnitude of the electric field due to the applied bias voltage $\left(V_{\text {bias }}\right)$, as illustrated in Fig. 1. When the TFT is nonconducting, the electric field across the photodiode gradually decreases due to the

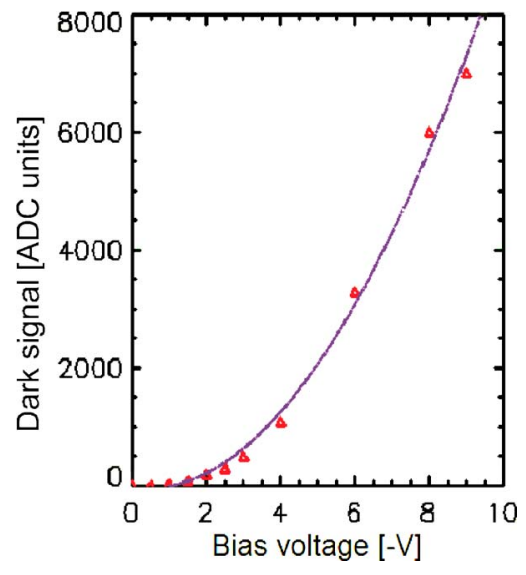

FIG. 1. Pixel dark signal in ADC units as a function of the applied bias voltage across the photodiode. This representative pixel was located near the center of the AMFPD. The signals were obtained for a fixed frame time at different bias voltages. The dark current can be obtained by dividing the dark charge (which is proportional to the dark signal) by the frame time. (Strictly speaking, this is only valid as the frame time approaches zero.) The electric field is proportional to the bias voltage given that the thickness of the photodiode pixel is constant. This graph shows that dark current increases rapidly as the electric field increases.

accumulation of charge originating from either dark current or radiation interactions. In general, the dark current is not constant due to the decrease of the electric field across the photodiode. On the other hand, pixel sensitivity, i.e., the amount of charge per unit dose due to radiation, also depends on the strength of the electric field across the photodiode (Sec. III A), and how it varies during irradiation. In general, pixel sensitivity and dark current depend on the electric field and thus are dependent on the total charge generated by the radiation and the dark current. As charge accumulates during irradiation, the electric field gradually decreases leading to reduced dark current and reduced pixel sensitivity. The charge originating from radiation in a time $T_{\text {frame }}$ is $Q_{\text {rad }}$

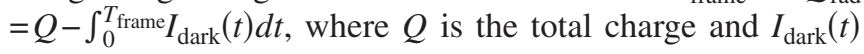
is the dark current. In the case of radiographic mode where the irradiation time can last for tens of seconds, the charge accumulated due to the dark current can be significantly different from that without radiation. Therefore, a correction based on the subtraction of a dark image acquired in the absence of radiation may be inaccurate and can lead to a dependence on the delivery-history of the resulting corrected image.

To illustrate this problem, Fig. 2 shows how the dark current may decrease during a period corresponding to the frame time $T_{\text {frame }}$ due to a decrease in the electric field. Since this decrease is hard to quantify due to its dependence on many factors, including the manner in which the radiation is delivered, it is difficult to accurately determine the dark signal contribution during $T_{\text {frame }}$. The removal of dark signal from the total radiation signal has thus far been performed by simply assuming a constant dark charge obtained in the absence of radiation. As illustrated in Fig. 2, the error from subtracting a constant dark charge during a frame time $T_{\text {frame }}$ is $\Delta Q_{1}+\Delta Q_{2}$. The error reduces to $\Delta Q_{1}$ if, for example, the 


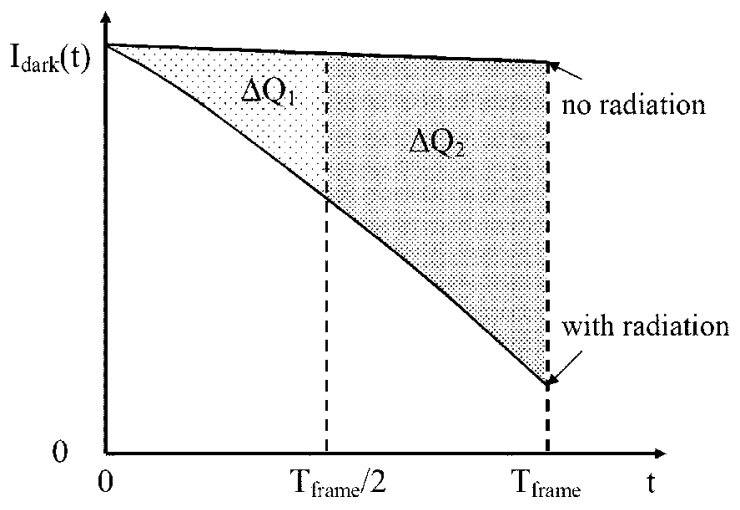

FIG. 2. A schematic graph showing dark current changes during irradiation. The upper straight line is the dark current in the absence of radiation during a time period $T_{\text {frame }}$ corresponding to the frame time. The lower curve is the dark current with radiation present. $\Delta Q_{1}$ is the error introduced by subtracting a constant dark charge during the first half of the frame time $T_{\text {frame }}$ and $\Delta Q_{2}$ is that error during the second half of the frame time.

frame time is halved. If the error proportionally decreases relative to the charge originating from irradiation, reducing the frame time cannot help to reduce the relative error. Fortunately this is not the case. Although the shape of $I_{\text {dark }}(t)$ curve can vary due to variations in the dose delivery, it is quite clear that $\Delta Q_{2}$ is greater than $\Delta Q_{1}$. If the dose rate at the pixel is constant, the charge generated by the radiation in the first half of a frame time equals that in the second half, i.e., $Q_{\text {rad1 }} \approx Q_{\text {rad2 }}$. Halving the frame time reduces the relative error due to dark current since $\Delta Q_{1} / Q_{\mathrm{rad} 1}<\Delta Q_{2} / Q_{\mathrm{rad} 2}$. Actually $Q_{\mathrm{rad2}}$ would be less than $Q_{\mathrm{rad} 1}$ since the pixel sensitivity reduces as the electric field decreases, which further supports the above conclusion. In the region of $I_{\text {dark }}(t)$ where $t$ $\rightarrow 0$, halving the frame time can reduce the relative error by a factor of two because $\left(\Delta Q_{1}+\Delta Q_{2}\right) /\left(Q_{\mathrm{rad} 1}+Q_{\mathrm{rad} 2}\right)$ $\approx 2\left(\Delta Q_{1} / Q_{\text {rad1 }}\right)$ as $t \rightarrow 0$. Given that frame times as long as tens of seconds can be necessary in radiographic mode in

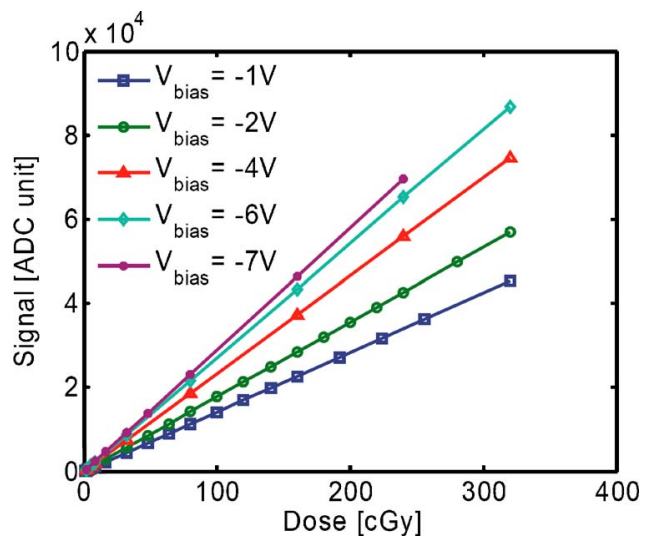

FIG. 3. Pixel dose response at different bias voltages. The AMFPD was operated in fluoroscopic mode with a frame time of $1.2 \mathrm{~s} .10 \times 10 \mathrm{~cm}^{2}$ open fields were measured at $90 \mathrm{~cm} \mathrm{SSD}$ and $10 \mathrm{~cm}$ depth in solid water phantom. The AMFPD signals from the central $10 \times 10$ pixels were averaged and then plotted in this graph. The absolute dose values were measured with an ion chamber in a water tank. The data were taken at a dose rate of 320 $\mathrm{cGy} / \mathrm{min}$ for $6 \mathrm{MV}$ photon beams. The linear correlation coefficient squared, $r^{2}$, for every response curve is greater than 0.999997 .

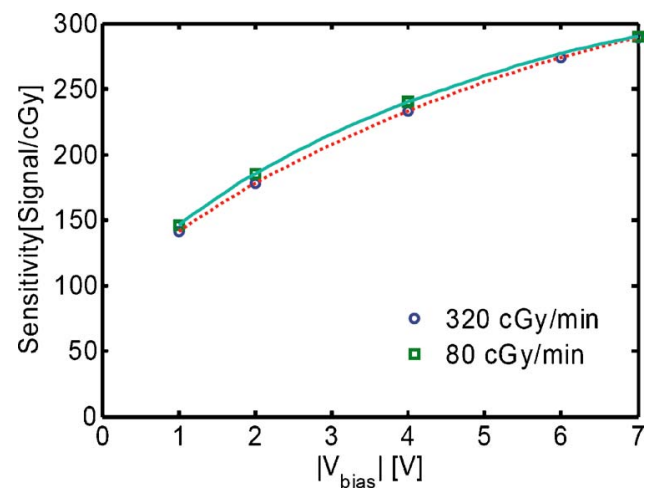

FIG. 4. Pixel sensitivity plotted as a function of bias voltage. The AMFPD was operated in fluoroscopic mode with a frame time of $1.2 \mathrm{~s}$. Several 10 $\times 10 \mathrm{~cm}^{2}$ open fields with various MUs were measured at bias voltages of $-1,-2,-4,-6$, and $-7 \mathrm{~V}$. The signals from the central $10 \times 10$ pixels were averaged, and the average pixel sensitivity was obtained. The data were taken at dose rates of $320 \mathrm{cGy} / \mathrm{min}$ and $80 \mathrm{cGy} / \mathrm{min}$ for $6 \mathrm{MV}$ photon beams.

order to accommodate the large doses delivered in a single treatment, and given that larger frame times result in increasingly less accurate dose estimation, it is advantageous to operate the AMFPD in fluoroscopic mode using considerably shorter frame times. In this manner, the total dose is spread over many frames as opposed to a single frame in radiographic mode. Therefore, the relative error due to constant dark charge subtraction is significantly reduced.

The fastest frame time allowed by the present AMFPD is $1.2 \mathrm{~s}$ in fluoroscopic mode. ${ }^{10}$ In this interval, the dose received in each frame is typically less than $10 \mathrm{cGy}$. Thus the corresponding change in the electric field is generally much smaller than that in the radiographic mode, leading to reduced variation in dark charge and better linearity in dose response.

In the present study, a fluoroscopic acquisition sequence used to measure a radiation field consisted of 30-100 ignored frames at the beginning of the acquisition before the radia-

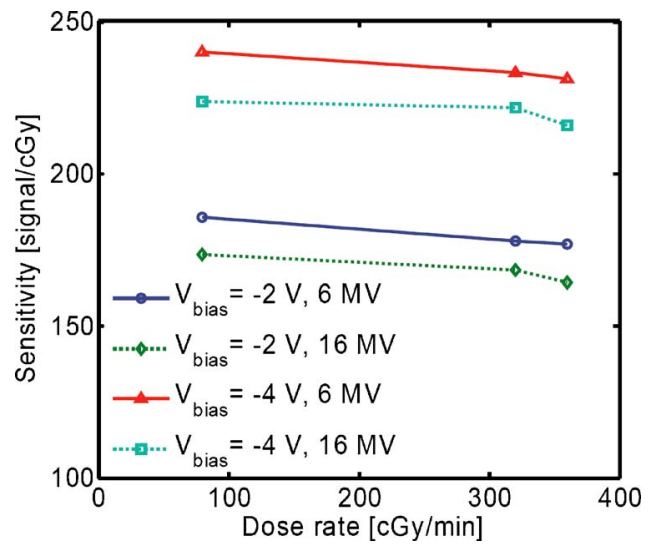

FIG. 5. Pixel sensitivity plotted as a function of dose rate. The AMFPD was operated in fluoroscopic mode with a frame time of $1.2 \mathrm{~s}$. Several 10 $\times 10 \mathrm{~cm}^{2}$ open fields with various MUs were measured at bias voltages of $-2 \mathrm{~V}$ and $-4 \mathrm{~V}$. The signals from the central $10 \times 10$ pixels were averaged, and the average pixel sensitivity was obtained. The data were taken at dose rates of 80,320 , and $360 \mathrm{cGy} / \mathrm{min}$ for $6 \mathrm{MV}$ and $16 \mathrm{MV}$ photon beams. 
tion begins (0.6-2 min), then 10-20 dark image frames followed by the data image frames obtained during the irradiation, and finally 20-30 image frames after the radiation stops (to measure the lag). Ignored frames are performed before radiation is delivered in order to initialize the pixels to remove previously accumulated or trapped charge. The lag image frames are acquired to ensure that trapped charge released after the irradiation is measured. The acquisition electronics send a signal at the end of the dark image frames to inform the operator to start radiation delivery. When an acquisition sequence is finished, the average dark image frame is determined and subtracted from each of the data and lag image frames. Then the resulting net signal frames are summed. Finally, bad pixels are corrected through interpolation, and a pixel-by-pixel gain correction, obtained from a flood field irradiation, is applied. In order to obtain a relatively uniform field for the flood field, the AMFPD is placed on or near the floor perpendicular to the gantry in the solid water phantom at $10 \mathrm{~cm}$ depth. An algorithm is then used to preserve the remaining shape of the open field dose distribution at that distance and depth.

\section{II.C. Dose response linearity}

Dose response, as indicated by pixel sensitivity, was measured with static $10 \times 10 \mathrm{~cm}^{2}$ fields at $10 \mathrm{~cm}$ depth and 90 $\mathrm{cm}$ source-to-surface distance (SSD) for 6 and $16 \mathrm{MV}$ at dose rates ranging from 100 to $600 \mathrm{MU} / \mathrm{min}$ (in $100 \mathrm{MU} / \mathrm{min}$ increments) and at various $V_{\text {bias }}$ values ranging from -1 to $-7 \mathrm{~V}$. The 6 and $16 \mathrm{MV}$ photon beams were calibrated such that 1 MU delivers $0.8 \mathrm{cGy}$ of dose for a $10 \times 10 \mathrm{~cm}^{2}$ jaw field at $10 \mathrm{~cm}$ depth in water with $90 \mathrm{~cm}$ SSD. For a specific bias voltage, beam energy, and dose rate, images were acquired for different MU values, and an ion chamber point measurement was taken under the same geometric and irradiation conditions to get the absolute dose. The measured dose calibration curve was fit with a linear relation where the slope yielded the pixel sensitivity, and absolute pixel dose was obtained by multiplying the net pixel signal value and the appropriate pixel sensitivity.

\section{II.D. IMRT measurements}

Measurements were performed using a Varian 21 EX accelerator equipped with a 120 leaf multileaf collimator (Varian Medical Systems, Palo Alto, CA). The surface of the detector array was placed at 3, 5, and $10 \mathrm{~cm}$ depths for different IMRT measurements. The same IMRT fields were measured with XV or EDR2 film (depending on the maximum field dose). A film characteristic curve was obtained for each set of film measurements so that optical densities could be converted to dose. Processor response was monitored for a sampling of films using a sensitometer in each processing session. Fiducials (pinholes) were used to mark the central axes of the film for IMRT measurements. As described in Sec. II C, AMFPD measurements were independently converted to dose as well. Comparisons were made between the two-dimensional dose distributions measured with the AMFPD and with film.

The test cases shown in this article included prostate SMLC IMRT fields with $0.5 \times 0.5 \mathrm{~cm}^{2}$ beamlets, head and neck SMLC IMRT fields with $1 \times 1 \mathrm{~cm}^{2}$ beamlets, and high dose per fraction partial breast IMRT fields with 0.5 $\times 0.5 \mathrm{~cm}^{2}$ or $1 \times 1 \mathrm{~cm}^{2}$ beamlets sequenced and delivered with SMLC or DMLC IMRT. A dose rate of $400 \mathrm{MU} / \mathrm{min}$ (320 cGy/min measured at $10 \mathrm{~cm}$ depth and $90 \mathrm{~cm}$ SSD in water) was used for all deliveries. Measurements were taken at $5 \mathrm{~cm}$ depth unless otherwise specified.

\section{II.E. Data evaluation}

The dose distributions of individual fields were compared for the AMFPD and film using isodose overlays, dose difference evaluation, and extraction of sample profiles across regions of interest (ROIs). Also, to quantify the agreement between the AMFPD and film, we calculated $\gamma, \chi$, and dosegradient compensation (C) indices for all fields. ${ }^{11-13}$ The definitions of these quantities are as follows:

$$
\begin{aligned}
& \gamma\left(\vec{r}_{r}\right)=\min \left\{\sqrt{\frac{\left|\vec{r}_{c}-\vec{r}_{r}\right|^{2}}{\Delta r_{\mathrm{tol}}^{2}}+\frac{\left(D_{c}-D_{r}\right)^{2}}{\Delta D_{\mathrm{tol}}^{2}}} \text { for any } \vec{r}_{c}\right\}, \\
& \chi\left(\vec{r}_{r}\right)=\frac{D_{c}-D_{r}}{\sqrt{\Delta D_{\mathrm{tol}}^{2}+\Delta r_{\mathrm{tol}}^{2} \times\left|\nabla D_{r}\right|^{2}}},
\end{aligned}
$$

$$
C\left(\vec{r}_{r}\right)=\left\{\begin{array}{cc}
\frac{D_{c}-D_{r}}{\left|D_{c}-D_{r}\right|} \times \frac{\left|D_{c}-D_{r}\right|-G_{r} \times \Delta r_{\mathrm{tol}}}{\Delta D_{\mathrm{tol}},} & \text { if }\left|D_{c}-D_{r}\right|>G_{r} \times \Delta r_{\mathrm{tol}} \\
0, & \text { if }\left|D_{c}-D_{r}\right| \leq G_{r} \times \Delta r_{\mathrm{tol}}
\end{array},\right.
$$



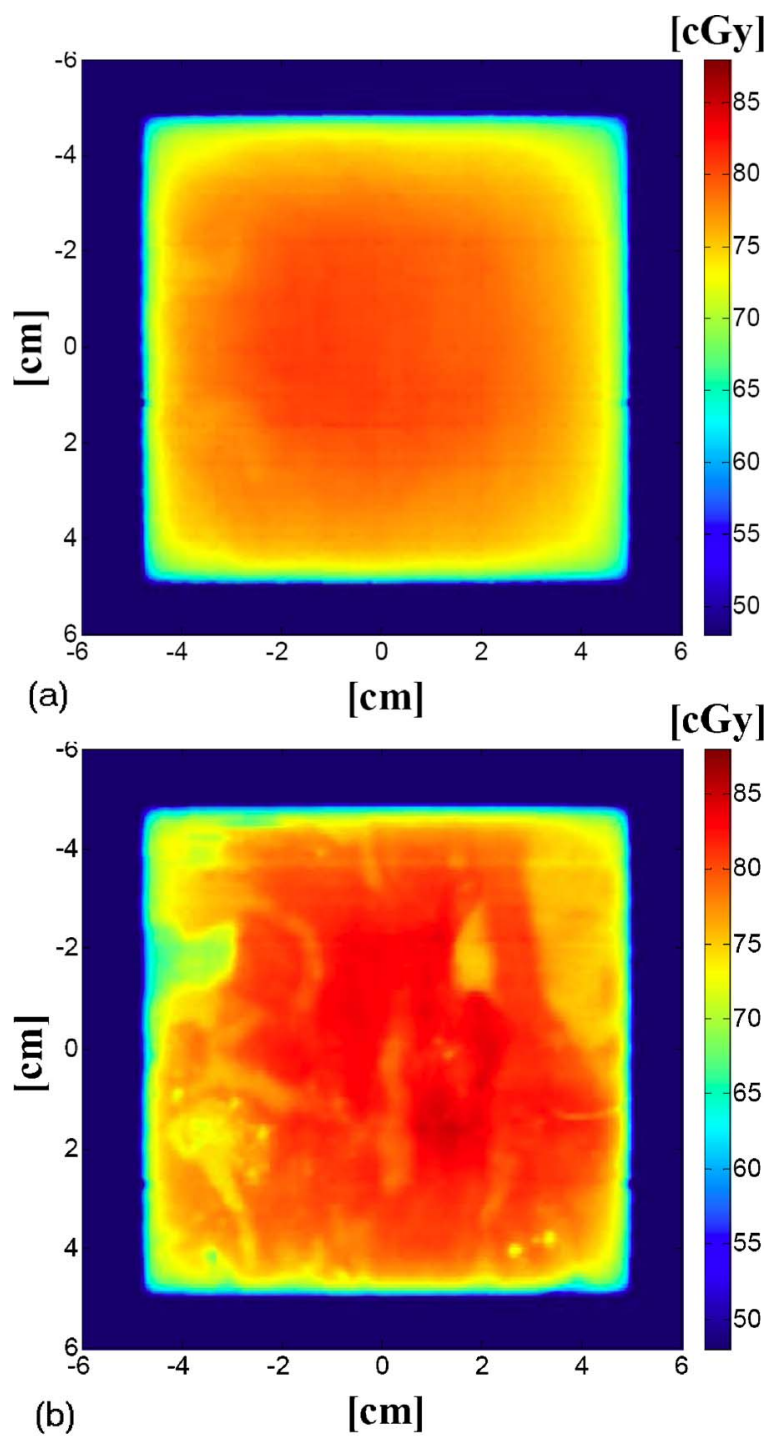

(b)
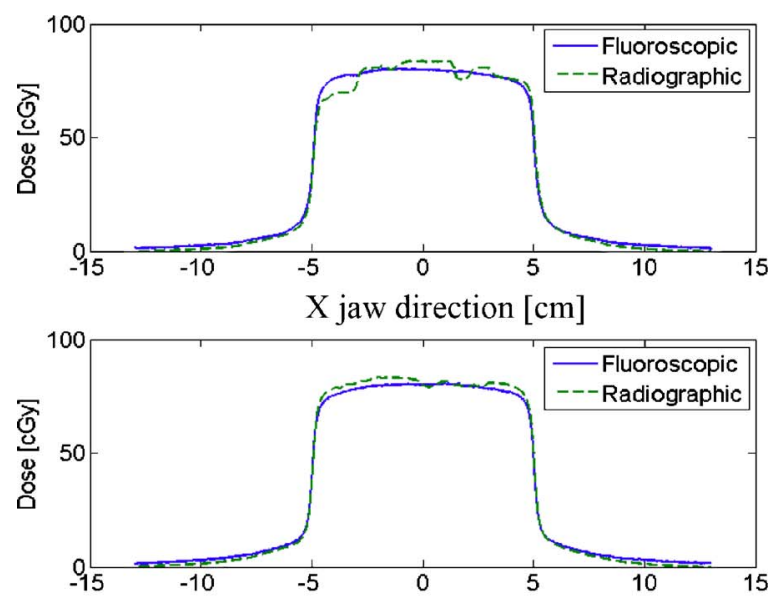

(c)

$\mathrm{Y}$ jaw direction $[\mathrm{cm}]$

FIG. 6. Dose response from a static $10 \times 10 \mathrm{~cm}^{2}$ field of $80 \mathrm{cGy}$ obtained with the AMFPD for a $6 \mathrm{MV}$ photon beam at a bias voltage of $-6 \mathrm{~V}$. (a) $2 \mathrm{D}$ dose response obtained in fluoroscopic mode with a frame time of $1.2 \mathrm{~s}$. (b) 2D dose response in radiographic mode with a frame time of $17 \mathrm{~s}$. (c) Sample line profiles extracted from (a) and (b) comparing fluoroscopic and radiographic acquisition mode in $X$ and $Y$ jaw directions. where $D_{c}$ is the dose map to be compared, $D_{r}$ is the reference dose map, $\vec{r}_{c}$ and $\vec{r}_{r}$ are the corresponding locations, $\Delta D_{\text {tol }}$ is the tolerance dose difference, and $\Delta r_{\text {tol }}$ is the distance to agreement (DTA). $G$ is defined as $G_{i}=\sqrt{\sum_{j}\left(\Delta D_{i j} / \Delta r_{i j}\right)^{2}}$, where $j$ loops around the nearest neighbors of $i$. $G$ is approximately as large as $\sqrt{2} \cdot|\nabla D|$ for a two-dimensional dose map.

The tolerance dose for the dose difference calculation was set to $2 \%$ of $D_{\max }$. The criteria of $2 \%$ of $D_{\max }\left(\Delta D_{\text {tol }}\right)$ and 2 mm DTA $\left(\Delta r_{\text {tol }}\right)$ (i.e., $2 \% / 2 \mathrm{~mm}$ ) were used to calculate the $\gamma$ and $\chi$ values. The $\chi$ and $C$ values for $2 \% / 1 \mathrm{~mm}$ criteria were also reported. To remove the bias on the level of agreement for very low dose regions, the ROI was determined as the area where the dose was higher than $5 \%$ of the maximum dose. A satisfaction index is defined as the percentage of the area within the ROI where certain criteria are satisfied over the total area of the ROI:

Satisfaction index

$$
=\frac{\# \text { of pixels whose }|\chi| \leq 1 \text { and } D>5 \% D_{\max }}{\# \text { of pixels whose } D>5 \% D_{\max }},
$$

where $\chi$ can be replaced by $\gamma$ or $C$ to get their respective satisfaction indices.

\section{RESULTS}

\section{III.A. Dose response linearity}

Dose response is characterized by the pixel sensitivity. The response in fluoroscopic mode with a $1.2 \mathrm{~s}$ frame time was linear for all situations (with correlation coefficient squared $r^{2}>0.99999$ ), as shown in Fig. 3. The highest dose tested in the present study was $1040 \mathrm{cGy}$, and the linear correlation coefficient square equals 0.999998 in this case. However, the pixel sensitivity varies with bias voltage and dose rate as shown in Figs. 4 and 5. Figure 4 shows that the pixel sensitivity decreases faster as the reverse bias voltage is reduced. Since the charge accumulated in the photodiode gradually decreases the electric field, the effective bias voltage across it would also decrease. For this reason, pixel sensitivity during the irradiation is not constant, leading to the nonlinear response in radiographic mode in the high dose region described in Sec. II B. Thus it is desirable to operate the AMFPD at a higher bias voltage where the pixel sensitivity decreases slowly as the electric field drops during irradiation.

For a fixed frame time in fluoroscopic mode, the pixel sensitivity also decreases slightly as the dose rate increases (Fig. 5). As the dose rate increases, the charge accumulated during one frame time increases, resulting in a slight decrease in the electric field across the photodiode, which in turn results in the dose rate dependence of the fluoroscopic mode. When compared with an ion chamber, measurements with the AMFPD in fluoroscopic mode have shown a higher increase in response with increasing field size. These differences can be corrected by deconvolving the raw signal with a kernel to account for low-energy photons. Since film has a 


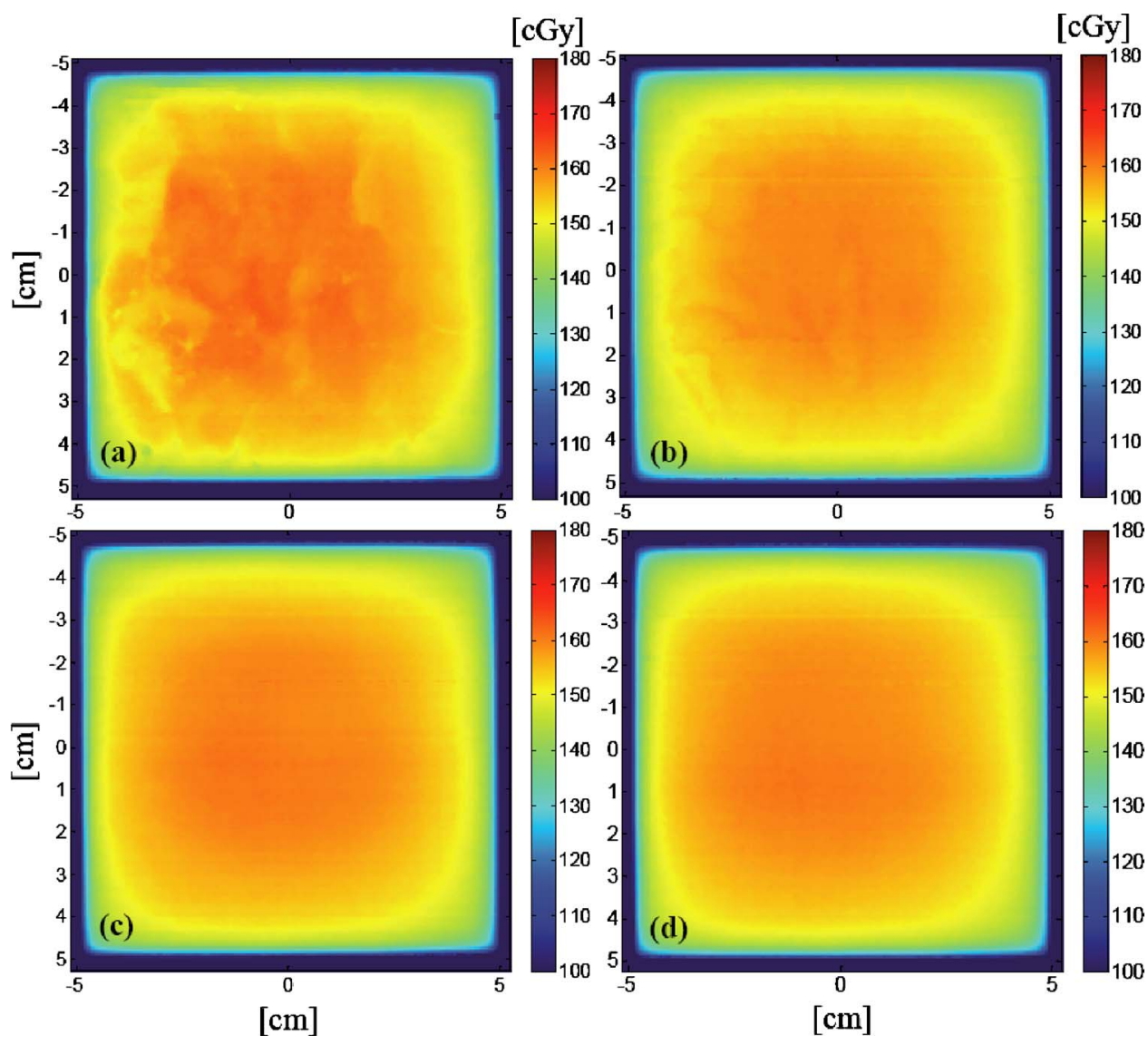

FIG. 7. Dose response from a static $10 \times 10 \mathrm{~cm}^{2}$ field of $160 \mathrm{cGy}$ obtained with the AMFPD in fluoroscopic mode with a frame time of $1.2 \mathrm{~s}$ for a $6 \mathrm{MV}$ photon beam at different bias voltages: (a) $-7 \mathrm{~V}$, (b) $-6 \mathrm{~V}$, (c) $-4 \mathrm{~V}$, and (d) $-2 \mathrm{~V}$. The plotted dose range is from 100 to $180 \mathrm{cGy}$. similar over-response, no additional corrections were made in this study for these effects to film or AMFPD data.

\section{III.B. Effect of dark current}

Static $10 \times 10 \mathrm{~cm}^{2}$ field images for $100 \mathrm{MU}$ were taken with the AMFPD at a $V_{\text {bias }}$ of $-6 \mathrm{~V}$ in both fluoroscopic and radiographic mode. The effect of dark current was significantly reduced in fluoroscopic mode compared with that in radiographic mode as shown in Fig. 6. In this particular case, the frame time of fluoroscopic mode was $1.2 \mathrm{~s}$ compared with $17 \mathrm{~s}$ of radiographic mode. Clearly, the radiation profile of the AMFPD in radiographic mode exhibits large discrepancies as compared with the dose profile one would expect to obtain from an ionization chamber measurement.

In fluoroscopic mode, static $10 \times 10 \mathrm{~cm}^{2}$ field images for the same amount of dose were taken at different bias voltages, as shown in Fig. 7. The change in the dark current still had a detectable effect on the dose image, as explained in the next paragraph, even though the effect was greatly reduced in comparison with the effect with radiographic mode.

A dose-independent pixel gain correction is insufficient to eliminate this effect because of the nonlinear relation between the dark current and the electric field. Ideally, the charge originating from radiation can be obtained by subtracting the dark charge from the total charge, i.e., $Q_{\mathrm{rad}}=Q$ $-Q_{\text {dark }}$. The actual pixel sensitivity is $\tilde{s}=\left(Q-Q_{\text {dark }}\right) / D$. However, the measured sensitivity is $s=\left(Q-Q_{\text {dark } 0}\right) / D$ where $Q_{\text {dark } 0}$ is measured in the absence of radiation. This leads to a problem in the pixel gain correction. Each pixel has a slightly different gain and dark current originating from the manufacturing process and from channel-to-channel variations in the preamplifiers. If one takes a pixel (No. 1) as the reference pixel and a second pixel (No. 2) is corrected relative to it, then the applied pixel gain correction can be expressed as $s_{2}=(1+\delta) s_{1}$, although, ideally, it should be $\tilde{s}_{2}$ $=(1+\theta) \widetilde{s}_{1}$. Here $\delta$ and $\theta$ represent the small variation in pixel sensitivity between the two photodiodes. Given that the actual pixel sensitivity, $\widetilde{s}$, is independent of dose when dose is small, $\theta$ can be assumed dose independent. Therefore, the gain would be corrected as long as the ratio $(1+\delta) /(1+\theta)$ is independent of dose. Since both $\delta$ and $\theta$ are small, the ratio is approximately $1+\delta-\theta$. It can readily be shown that

$$
\delta-\theta \approx \frac{\left(Q_{\text {dark }, 2}-Q_{\text {dark }, 1}\right)-\left(Q_{\text {dark } 0,2}-Q_{\text {dark } 0,1}\right)}{Q_{1}-Q_{\text {dark }, 1}} .
$$

The quantity $\delta-\theta$ may not be constant with respect to dose since the quantity related to dark current, $\left(Q_{\text {dark,2 }}\right.$ $\left.-Q_{\text {dark, } 1}\right)-\left(Q_{\text {dark } 0,2}-Q_{\text {dark } 0,1}\right)$, is not, in general, linear with dose. Although the pixel gain was corrected at a certain dose per frame with a flood field, the difference between different pixels in the corrected image could still be detectable at another dose per frame due to changes in dark current. This is a result of the dose-independent dark charge subtraction and dose-independent pixel gain correction. However, the dark current decreases much faster with a reduced bias voltage than the charge signal $Q_{1}-Q_{\text {dark,1 }}$. Therefore, when the bias 


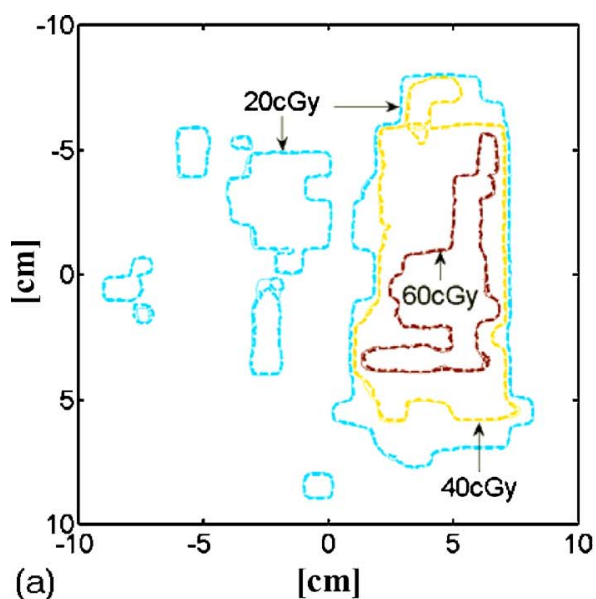

(c)

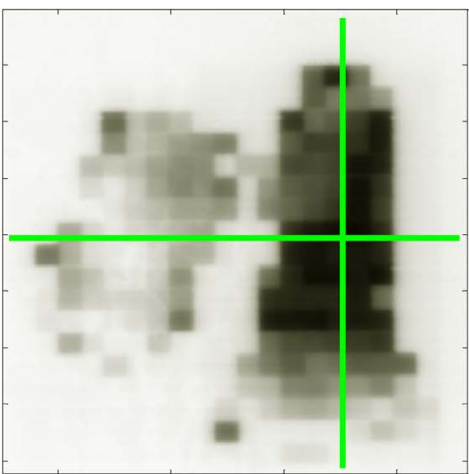

(a)

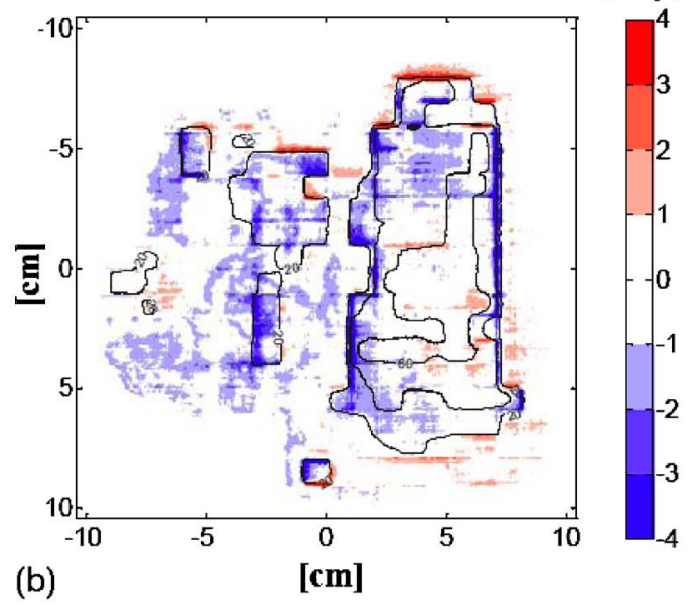

[cGy]
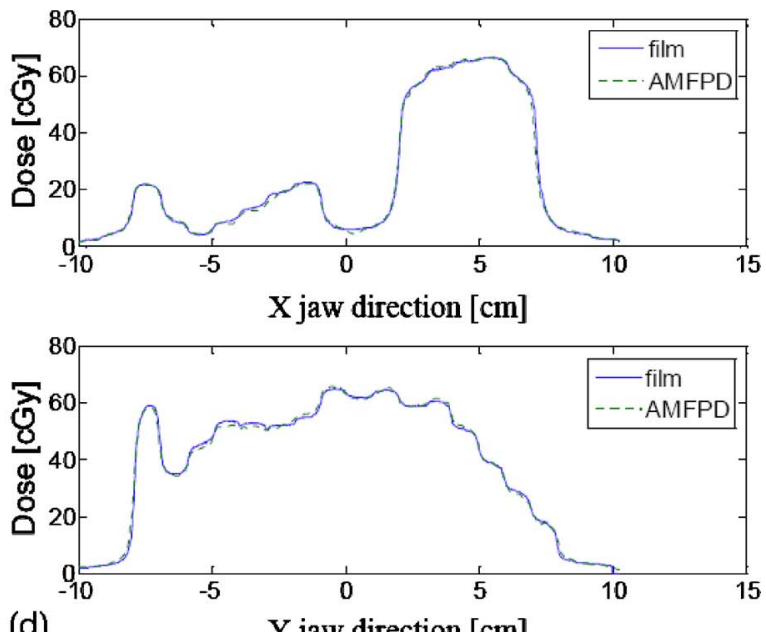

(d)

$\mathrm{Y}$ jaw direction $[\mathrm{cm}]$

FIG. 8. Results corresponding to head and neck case B1: (a) iso-dose overlay plot obtained with AMFPD (dashed line) and film (solid line); (b) dose difference plot in cGy (AMFPD-film); (c) AMFPD dose map; and (d) sample line profile comparison between the AMFPD and film along the lines as indicated in (c). For these results, and that shown in Figs. 9 and 10, the AMFPD was operated in fluoroscopic mode with a frame time of $1.2 \mathrm{~s}$.

voltage is low, the quantity related to dark charge is much less than the charge generated by the radiation. Thus, the impact of the change in the dark current is not as large as when the bias voltage is high.

The above considerations provide motivation to operate the AMFPD at a low bias voltage. However, as discussed in Sec. III A, a consideration of the dependence of the pixel sensitivity on the bias voltage favors a higher bias voltage. Thus, a compromise must be made in choosing the optimal bias voltage. We found that a bias voltage from $-4 \mathrm{~V}$ to $-6 \mathrm{~V}$ was suitable for reliable dosimetry with a frame time of $1.2 \mathrm{~s}$. The bias voltage used in the following IMRT measurements was $-6 \mathrm{~V}$ (prostate $B 1 \sim B 7, \mathrm{H} \& \mathrm{~N} B 1 \sim B 9$, PBI $B 1 \sim B 3$ ), and $-4 \mathrm{~V}$ (remaining cases).

\section{III.C. IMRT measurements}

For illustration, the results for three sample fields are shown in Figs. 8-10. For each field, isodose overlays, dose difference plots, dose maps, and sample line profiles are shown. Figure 8 shows a head and neck field (H\&N B1 in Table I) composed of $1 \times 1 \mathrm{~cm}^{2}$ beamlets. It consists of two split fields overlapping for $3 \mathrm{~cm}$. The first field has $95 \mathrm{seg}$ - ments, and the second field has 79 segments. The two images were combined after the SMLC deliveries. Figure 9 shows a partial breast field (DMLC PBI $3 \mathrm{~cm}$ in Table I) with 1 $\times 1 \mathrm{~cm}^{2}$ beamlets. This field has 150 control points and was delivered with DMLC. The AMFPD was placed at a $3 \mathrm{~cm}$ depth for this field. Figure 10 shows a prostate field (Prostate B7 in Table I) composed of $0.5 \times 0.5 \mathrm{~cm}^{2}$ beamlets and SMLC delivery consisting of 83 segments. Two line profiles across the high dose region were extracted for each field.

As described in Sec. II E, the satisfaction indices of $\gamma, \chi$, and $C$ were calculated with various criteria, along with the number of segments and the maximum dose for each field, shown in Table I. Table II demonstrates the stability of the AMFPD. When AMFPD measurements on different dates were compared, nearly $100 \%$ of the ROI satisfies the 2\%/2 mm criteria. When XV film measurements taken on different dates were compared, the results showed similar excellent consistency (Table III), though agreement between EDR2 and XV was much worse. The agreement between the AMFPD and XV was only slightly worse than the demonstrated XV consistency (see the bottom row in Table III). As 


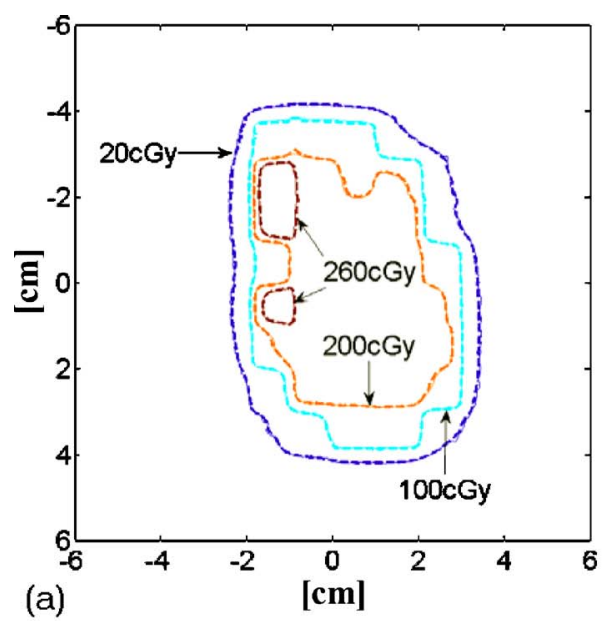

(c)

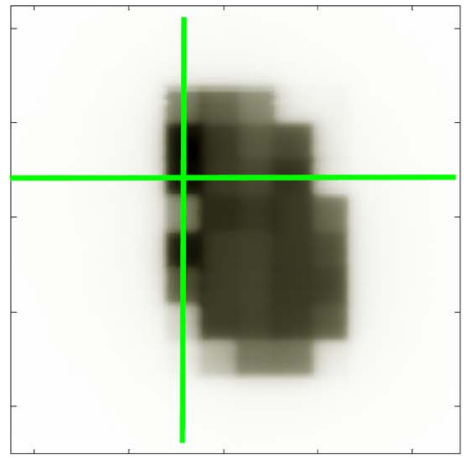

(a)
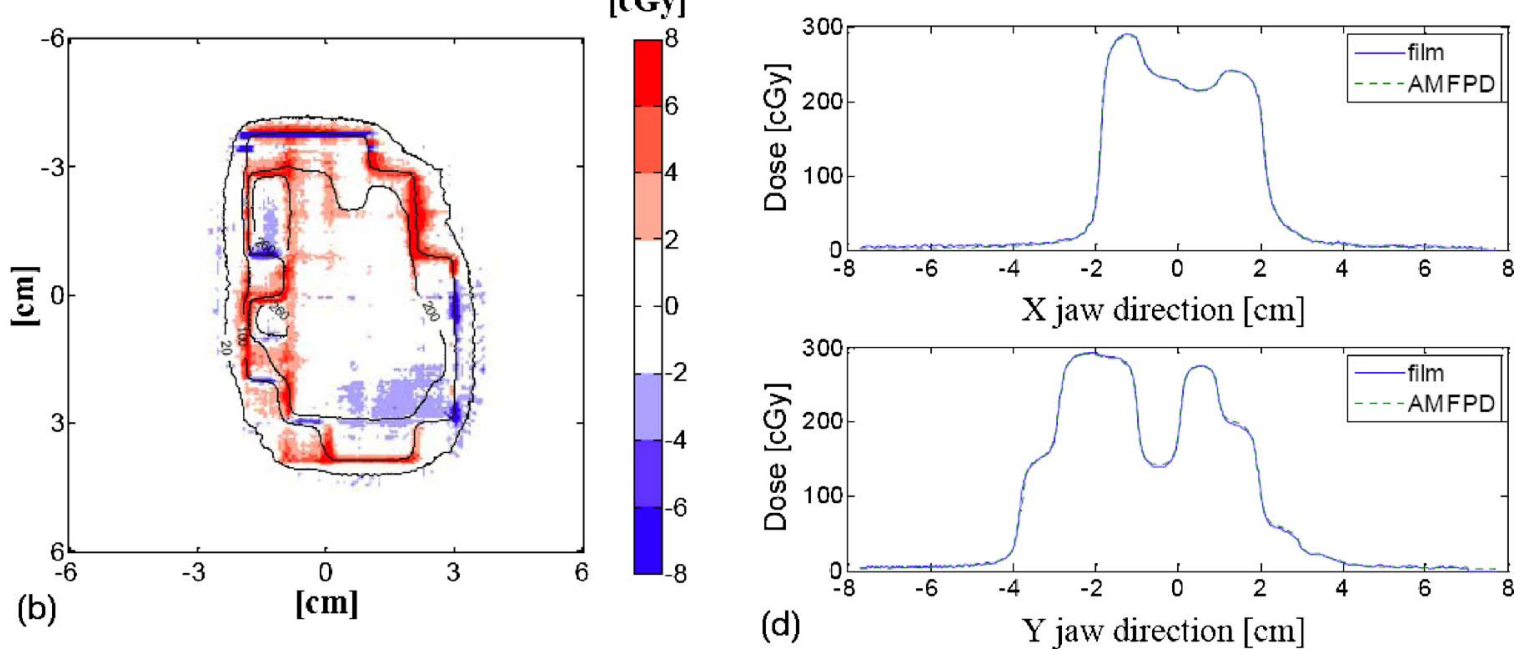

FIG. 9. Results corresponding to DMLC PBI case at a depth of $3 \mathrm{~cm}$ : (a) iso-dose overlay plot obtained with AMFPD (dashed line) and film (solid line); (b) dose difference plot in cGy (AMFPD-film); (c) AMFPD dose map; and (d) sample line profile comparison between the AMFPD and film along the lines as indicated in (c).

noted in case 3 of Table III, the radiation field was delivered twice for the EDR2 film in order to achieve an adequate signal-to-noise ratio for the low dose field.

A series of $\mathrm{XV}$ film data in dose response (H\&D curve) measurements shows that the relative error $(\sigma /$ mean) due to random noise is less than $0.7 \%$ at the isocenter for all the 19 fields with dose from 0 to $192 \mathrm{cGy}$. The difference between dose measured with film and that with an ion chamber for the same series of XV film data is $0.0 \pm 0.5 \mathrm{cGy}$.

\section{DISCUSSION}

\section{IV.A. Dose response linearity}

As reported in our previous work, ${ }^{8}$ the dose response of each pixel is not linear in radiographic mode, especially when the dose is high. Clearly, the intrinsic nonlinear dose response for a single frame time is due to the decrease in the electric field across the photodiode as more and more charge is accumulated and the pixel sensitivity is no longer a constant.

The AMFPD demonstrates excellent linearity of the dose response in fluoroscopic mode. This is because the dose de- livered to the AMFPD is divided into many frames by readout of the signal at a constant rate. Even though the dose response might not be linear for a given fraction in a single frame time, the overall response is still linear as long as the frame time is much smaller than the total delivery time. Also, the total amount of dose that can be delivered to a pixel is limited only by the memory size of the control computer to store all the frames. In this work, we found excellent linearity for doses up to $1040 \mathrm{cGy}$.

The bias voltage dependence of the pixel sensitivity for direct detection is different from that for indirect detection with a scintillator screen. ${ }^{10}$ For indirect detection, the $\mathrm{x}$ rays are converted into optical photons, and the absorption of these optical photons happens closer to the $p$-doped layer near the top of the photodiode. The signal transport mechanism is dominated by the transit of electrons through the intrinsic layer. Therefore, the charge collection efficiency does not change much for a wide range of the bias voltage, and thus the pixel sensitivity is almost constant. ${ }^{9,10}$ On the other hand, for direct detection the x-ray interactions are more uniformly distributed along the depth of the intrinsic $a-\mathrm{Si}: \mathrm{H}$ bulk. Therefore, more electron-hole pairs are gener- 


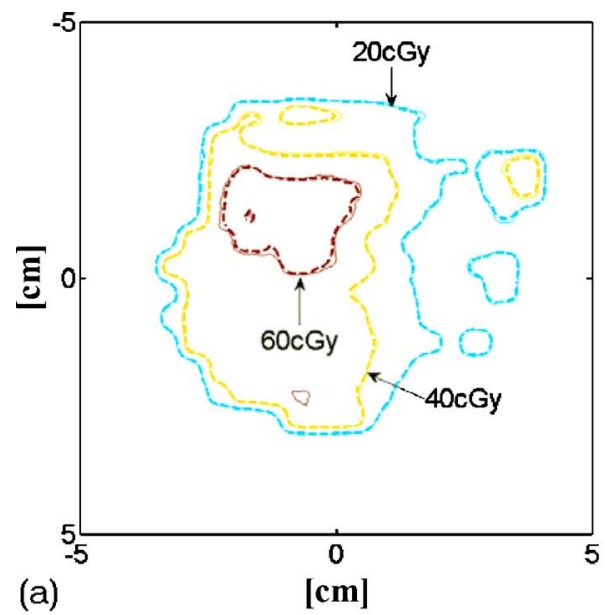

(c)

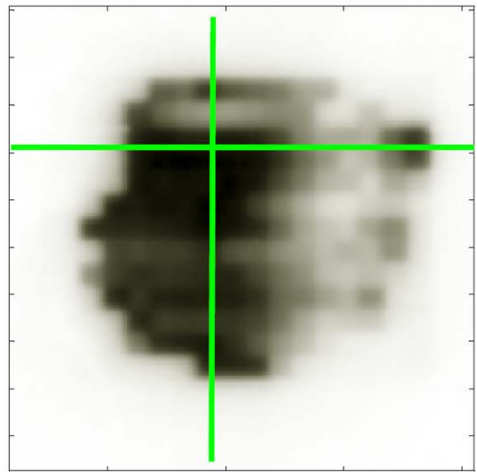

[cGy]

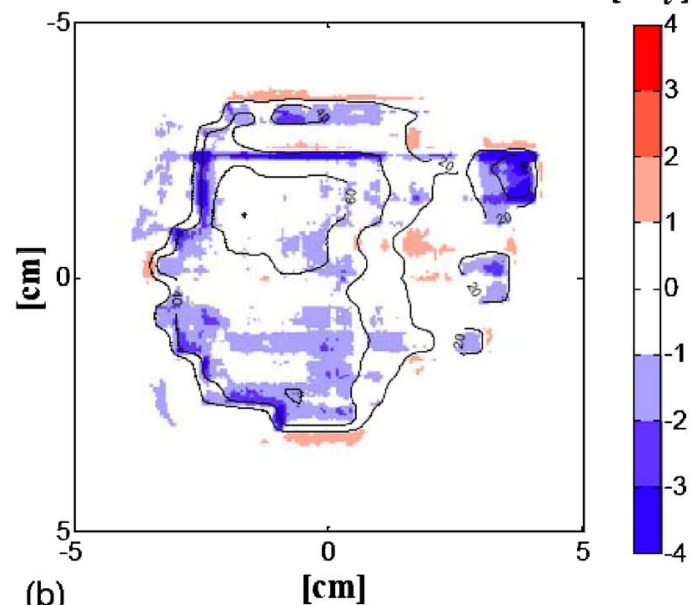

(b)

[cm]
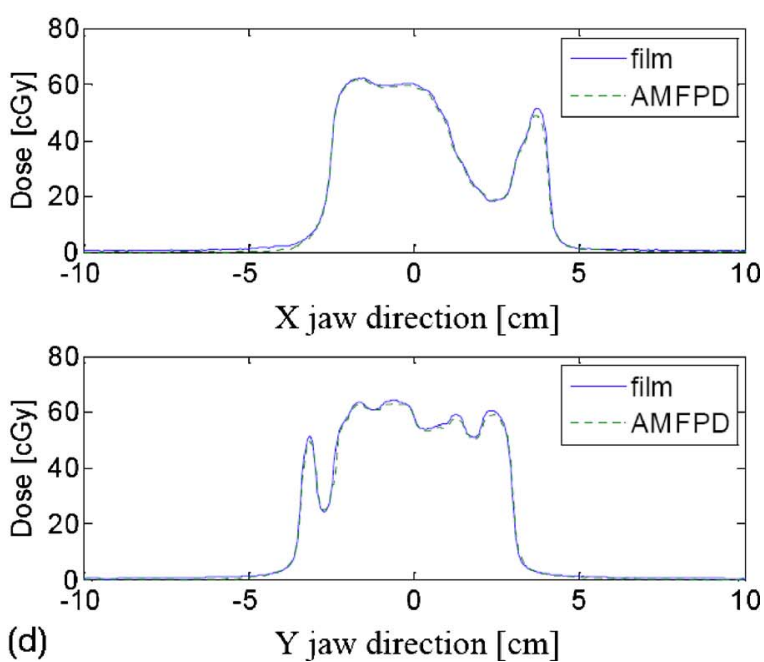

FIG. 10. Results corresponding to prostate case B7 with $0.5 \times 0.5 \mathrm{~cm}^{2}$ beamlets: (a) iso-dose overlay plot obtained with AMFPD (dashed line) and film (solid line); (b) dose difference plot in cGy (AMFPD-film); (c) AMFPD dose map; and (d) sample line profile comparison between the AMFPD and film along the lines as indicated in (c).

ated deeper in the intrinsic layer. In this case the signal transport mechanism is a combination of the transit of electrons and holes. Due to the much lower mobility of holes $\left(\sim 10^{-3} \mathrm{~cm}^{2} / \mathrm{V} \mathrm{s}\right)$ relative to that of electrons $\left(\sim 1 \mathrm{~cm}^{2} / \mathrm{Vs}\right)$, the recombination probability may be higher, and the charge collection may not be as effective as in the case of indirect detection and the bias voltage dependence would be stronger.

\section{IV.B. Dark current and acquisition mode considerations}

As seen in Fig. 7, the level of dark current affects the dose image quality, allowing better results at a lower bias voltage. However, the bias voltage dependence of the pixel sensitivity is less desirable for a lower bias voltage when the actual or effective dose rate varies, as may occur when beam hold offs are executed when delivering SMLC or DMLC fields. ${ }^{14}$ Another factor that affects the dose image quality is the signal to noise ratio (SNR). The dose response sensitivity is significantly lower if a low bias voltage is applied, which also jeopardizes the dose image quality. Therefore, these tradeoffs must be considered when choosing an appropriate bias voltage.

The dosimetric performance of the AMFPD can be improved by a higher frame rate. A shorter frame time makes the dose in each frame smaller. Hence, the dark charge and pixel sensitivity become more constant. Also, artifacts from the dose-independent pixel gain correction as well as dose rate dependence will be reduced. As the dark charge decreases, the AMFPD can be operated at a higher bias voltage to improve SNR. The initialization time for the ignored frames can be eliminated for a system whose acquisition electronics operate in a continuous readout mode whenever the system is powered on.

\section{IV.C. IMRT measurements}

In general, we found excellent agreement between the IMRT dose distributions of the AMFPD and that of both XV and EDR2 film (Table I). If $2 \% / 2 \mathrm{~mm}$ is taken as the criteria for agreement, on average, $97.5 \pm 1.9 \%$ of the region where the dose is greater than $5 \%$ of the maximum dose satisfies 
TABLE I. Results of comparison of AMFPD and film measurements made for 22 fields in this study. The values reported are the satisfaction indices using each of $\gamma, \chi$, and $C$, for different criteria. Measurements were taken at $5 \mathrm{~cm}$ depth for the first 19 fields. The last three fields were measured at depths of 10,10 , and $3 \mathrm{~cm}$, respectively. The number of segments for SMLC or control points for DMLC in each field is listed in the second column.

\begin{tabular}{|c|c|c|c|c|c|c|c|}
\hline Field criteria & Segments & $\begin{array}{l}D_{\max } \\
\text { (cGy) }\end{array}$ & $\begin{array}{c}\gamma \\
2 \% / 2 \mathrm{~mm}\end{array}$ & $2 \% / 0 \mathrm{~mm}$ & $\begin{array}{c}\chi \\
2 \% / 1 \mathrm{~mm}\end{array}$ & $2 \% / 2 \mathrm{~mm}$ & $\begin{array}{c}C \\
2 \% / 1 \mathrm{~mm}\end{array}$ \\
\hline Prostate B1 & 87 & 62 & $96.5 \%$ & $69.1 \%$ & $91.4 \%$ & $96.8 \%$ & $98.1 \%$ \\
\hline Prostate B2 & 75 & 62 & $97.7 \%$ & $74.4 \%$ & $94.8 \%$ & $98.1 \%$ & $99.2 \%$ \\
\hline Prostate B3 & 81 & 65 & $98.2 \%$ & $83.1 \%$ & $96.8 \%$ & $98.6 \%$ & $99.5 \%$ \\
\hline Prostate B4 & 95 & 58 & $97.4 \%$ & $69.6 \%$ & $95.6 \%$ & $98.8 \%$ & $99.5 \%$ \\
\hline Prostate B5 & 84 & 66 & $95.6 \%$ & $66.8 \%$ & $89.4 \%$ & $95.9 \%$ & $98.2 \%$ \\
\hline Prostate B6 & 71 & 62 & $95.5 \%$ & $75.9 \%$ & $93.1 \%$ & $96.5 \%$ & $98.0 \%$ \\
\hline Prostate B7 & 83 & 64 & $98.2 \%$ & $85.4 \%$ & $98.0 \%$ & $99.3 \%$ & $99.9 \%$ \\
\hline $\mathrm{H} \& \mathrm{~N}$ B1 & $95+79$ & 67 & $97.7 \%$ & $81.3 \%$ & $94.4 \%$ & $97.5 \%$ & $99.1 \%$ \\
\hline $\mathrm{H} \& \mathrm{~N}$ B2 & 73 & 39 & $95.8 \%$ & $76.2 \%$ & $90.3 \%$ & $94.6 \%$ & $98.1 \%$ \\
\hline $\mathrm{H} \& \mathrm{~N}$ B3 & $94+48$ & 56 & $99.1 \%$ & $94.8 \%$ & $98.9 \%$ & $99.4 \%$ & $99.8 \%$ \\
\hline $\mathrm{H} \& \mathrm{~N}$ B4 & $110+69$ & 52 & $97.9 \%$ & $86.8 \%$ & $96.0 \%$ & $98.1 \%$ & $99.5 \%$ \\
\hline H\&N B5 & $101+88$ & 63 & $92.4 \%$ & $71.1 \%$ & $86.7 \%$ & $92.3 \%$ & $95.4 \%$ \\
\hline $\mathrm{H} \& \mathrm{~N}$ B6 & $104+108$ & 65 & $97.2 \%$ & $86.3 \%$ & $94.9 \%$ & $97.0 \%$ & $98.8 \%$ \\
\hline $\mathrm{H} \& \mathrm{~N} B 7$ & 74 & 48 & $98.3 \%$ & $86.9 \%$ & $96.3 \%$ & $98.4 \%$ & $99.6 \%$ \\
\hline $\mathrm{H} \& \mathrm{~N}$ B8 & $106+119$ & 44 & $96.4 \%$ & $76.9 \%$ & $91.2 \%$ & $96.6 \%$ & $98.6 \%$ \\
\hline$H \& N$ B9 & $94+80$ & 53 & $98.3 \%$ & $87.8 \%$ & $96.3 \%$ & $98.2 \%$ & $99.5 \%$ \\
\hline PBI B1 & 95 & 172 & $96.2 \%$ & $74.2 \%$ & $91.6 \%$ & $96.5 \%$ & $98.6 \%$ \\
\hline PBI B2 & 87 & 198 & $94.9 \%$ & $73.1 \%$ & $99.8 \%$ & $94.9 \%$ & $98.0 \%$ \\
\hline PBI B3 & 82 & 223 & $99.2 \%$ & $91.4 \%$ & $98.3 \%$ & $99.4 \%$ & $100.0 \%$ \\
\hline $\mathrm{H} \& \mathrm{~N}$ B10 $10 \mathrm{~cm}$ & $127+149$ & 55 & $99.0 \%$ & $85.0 \%$ & $96.6 \%$ & $99.0 \%$ & $99.8 \%$ \\
\hline DMLC PBI $10 \mathrm{~cm}$ & 150 & 158 & $98.6 \%$ & $84.3 \%$ & $99.3 \%$ & $100.0 \%$ & $100.0 \%$ \\
\hline DMLC PBI $3 \mathrm{~cm}$ & 150 & 296 & $98.5 \%$ & $93.1 \%$ & $100.0 \%$ & $100.0 \%$ & $100.0 \%$ \\
\hline Mean \pm std & & & $97.2 \pm 1.7 \%$ & $80.6 \pm 8.2 \%$ & $94.5 \pm 3.7 \%$ & $97.5 \pm 1.9 \%$ & $98.9 \pm 1.1 \%$ \\
\hline
\end{tabular}

$|\chi| \leq 1$. As expected, evaluation based on $\gamma$ gave almost the same results as that based on $|\chi|$. Since the AMFPD and film were irradiated separately, variations in the delivery of the radiation might also lead to small differences between the dose distributions. The consistency of the AMFPD dose distributions for IMRT fields was verified by comparing dose images obtained on different dates (Table II). In this case, $99.9 \%$ of the region where dose is greater than $5 \%$ of maximum satisfies $|\chi| \leq 1$. This result is similar to that from comparisons of a single type of film and better than that from comparisons between different film types (Table III). This suggests that the AMFPD can be a substitute for film in IMRT dosimetry measurements since the discrepancy between different types of film is no less than that between an
AMFPD and film. For example, Dogan et al. also reported discrepancies between EDR2 and XV2 films for IMRT verification. $^{15}$

In the present study, the few discrepancies between the AMFPD and film measurements were primarily in regions where high dose is delivered to a small area (such as a single beamlet). The region of single beamlet spikes is often problematic for comparisons, and more research will be needed to determine which systems, analysis, or techniques are causing the discrepancies.

The comparison between the AMFPD and film was made for both SMLC and DMLC fields, with $0.5 \times 0.5 \mathrm{~cm}^{2}$ and $1 \times 1 \mathrm{~cm}^{2}$ beamlets, at different depths. These results show that the AMFPD is capable of IMRT QA verification as a

TABLE II. Comparisons of AMFPD measurements performed on different dates. The values reported are the satisfaction indices using each of $\gamma, \chi$, and $C$, for different criteria. Measurements were taken at $5 \mathrm{~cm}$ depth. The number of segments in each field is listed in the second column. This comparison indicates the consistency of the AMFPD.

\begin{tabular}{|c|c|c|c|c|c|c|c|}
\hline Field criteria & Segments & $\begin{array}{l}D_{\max } \\
(\mathrm{cGy})\end{array}$ & $\begin{array}{c}\gamma \\
2 \% / 2 \mathrm{~mm}\end{array}$ & $2 \% / 0 \mathrm{~mm}$ & $\begin{array}{c}\chi \\
2 \% / 1 \mathrm{~mm}\end{array}$ & $2 \% / 2 \mathrm{~mm}$ & $\begin{array}{c}C \\
2 \% / 1 \mathrm{~mm}\end{array}$ \\
\hline PBI B2 & 87 & 197 & $99.9 \%$ & $97.9 \%$ & $99.8 \%$ & $99.9 \%$ & $100.0 \%$ \\
\hline PBI B3 & 82 & 221 & $100.0 \%$ & $99.4 \%$ & $100.0 \%$ & $100.0 \%$ & $100.0 \%$ \\
\hline H\&N B1 & $95+79$ & 67 & $100.0 \%$ & $99.4 \%$ & $99.9 \%$ & $100.0 \%$ & $100.0 \%$ \\
\hline H\&N B7 & 74 & 48 & $99.9 \%$ & $98.5 \%$ & $99.7 \%$ & $99.8 \%$ & $100.0 \%$ \\
\hline
\end{tabular}


TABLE III. Comparisons of film measurements performed on different dates (case 1) or with different types of film (case 2). The values reported are the satisfaction indices using each of $\gamma, \chi$, and $C$, for different criteria. Measurements were taken at $10 \mathrm{~cm}$ depth for the same field of a head and neck case $(H \& N$ B10 $10 \mathrm{~cm})$. The number of segments in each field is listed in the second column.

\begin{tabular}{lccccrrr}
\hline \hline \multirow{2}{*}{ Case criteria } & \multirow{2}{*}{$\begin{array}{c}D_{\max } \\
(\mathrm{cGy})\end{array}$} & $\begin{array}{c}\gamma \\
2 \% / 2 \mathrm{~mm}\end{array}$ & $2 \% / 0 \mathrm{~mm}$ & $\begin{array}{c}\chi \\
2 \% / 1\end{array}$ & $2 \% / 2 \mathrm{~mm}$ & $\begin{array}{c}C \\
2 \% / 1 \mathrm{~mm}\end{array}$ \\
\hline 1: xv-xv & $127+149$ & 55 & $99.7 \%$ & $96.6 \%$ & $100.0 \%$ & $100.0 \%$ & $100.0 \%$ \\
2: edr-xv & $127+149$ & 55 & $91.6 \%$ & $67.6 \%$ & $82.1 \%$ & $90.0 \%$ & $95.8 \%$ \\
3: AMFPD-xv & $127+149$ & 55 & $99.0 \%$ & $85.0 \%$ & $96.6 \%$ & $99.0 \%$ & $99.8 \%$ \\
\hline \hline
\end{tabular}

substitute for film measurements. This system maintains excellent uniform spatial resolution $(0.508 \mathrm{~mm})$ over the entire field (up to $26 \times 26 \mathrm{~cm}^{2}$ ), compared with the typically nonuniform resolution (ranging from several millimeters to several centimeters) for available diode and ionization chamber array systems. ${ }^{2,3}$

\section{IV.D. Comparison to other verification systems}

Although the AMFPD system is based on the same type of $a-\mathrm{Si}: \mathrm{H}$ detector plate used within commercial EPID systems, there are a number of significant differences between IMRT dosimetry measurements made with the AMFPD system and IMRT verification methods based on commercial EPIDs. First, the AMFPD system is used in a solid water phantom so it implements dosimetry measurements in phantom, as most radiotherapy dosimetry is performed. For EPID-based IMRT field verification, investigators have developed algorithms to reconstruct the delivered fluence map from the portal image measured in the EPID. ${ }^{4,6,16}$ This reconstructed fluence map can then be calculationally convolved with a dose kernel to yield the expected two-dimensional dose distribution in water, or it can be applied to the patient geometry to calculate the three-dimensional dose distribution in the patient. For example, one algorithm utilizes the measured dose response kernel of the detector to calculate the portal dose in the detector given the intended fluence map. ${ }^{5}$ The calculated portal dose is then compared with the measured one. Another algorithm takes advantage of the symmetric geometry of the virtual phantom to derive the dose distribution in that phantom from the fluence-related portal image. ${ }^{17}$ All of these verification methods combine the output from the portal image (i.e., portal dose, detector dose response kernel, or reconstructed fluence) with an algorithm that always involves the use of treatment planning-like calculational information and/or methods (from the treatment planning system or an independent source of planning system-like information). This calculated result may be partially or totally independent of the clinical treatment planning system, but it is always based on a calculational method and is not a direct measurement of the dose distribution that has been delivered. Because the AMFPD system directly measures the dose in phantom, the AMFPD-based verification method is a much more robust and independent check on IMRT delivery than can be achieved with an EPID-based system.

\section{CONCLUSIONS}

The results reported in this article suggest that AMFPDs could serve as excellent electronic dosimeters for IMRT and could be used to replace film for phantom measurements. When operated in continuous fluoroscopic acquisition mode, the AMFPD characterized in this study maintained an excellent linear dose response up to $1040 \mathrm{cGy}$ over the six month period of the study. In order to obtain reliable integrated dose results for IMRT fields, the effects of the dark current on the radiation signal were minimized. Compared with film, the AMFPD results were excellent, generally within $2 \% / \pm 2 \mathrm{~mm}$. Compared with other electronic devices, the AMFPD has much higher spatial resolution $(0.508 \mathrm{~mm})$ across the entire measurement area of $26 \times 26 \mathrm{~cm}^{2}$ and provides high quality two-dimensional dosimetric measurements for IMRT delivery characterization and verification.

\section{ACKNOWLEDGMENTS}

We would like to thank Joann I. Prisciandaro and Sue Henshaw for providing some IMRT film data. The work was supported in part by NCI P01 CA59827 and the University of Michigan Cancer Center.

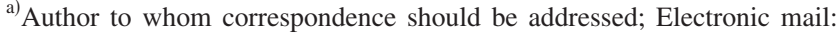
ychen7@gmail.com

${ }^{1}$ J. M. Galvin, G. Ezzell, A. Eisbrauch, C. Yu, B. Butler, Y. Xiao, I. Rosen, J. Rosenman, M. Sharpe, L. Xing, P. Xia, T. Lomax, D. A. Low, and J. Palta, "Implementing IMRT in clinical practice: a joint document of the American society for therapeutic radiology and oncology and the American Association of Physicists in Medicine," Int. J. Radiat. Oncol. Biol. Phys. 58, 1616-1634 (2004).

${ }^{2}$ D. Létourneau, M. Gulam, D. Yan, M. Oldham, and J. W. Wong, "Evaluation of a 2D diode array for IMRT quality assurance," Radiother. Oncol. 70, 199-206 (2004).

${ }^{3}$ E. Spezi, A. L. Angelini, F. Romani, and A. Ferri, "Characterization of a 2D ion chamber array for the verification of radiotherapy treatments," Phys. Med. Biol. 50, 3361-3373 (2005).

${ }^{4}$ B. B. Warkentin, S. Steciw, S. Rathee, and B. G. Fallone, "Dosimetric IMRT verification with a flat-panel EPID," Med. Phys. 30, 3143-3155 (2003).

${ }^{5}$ A. Van Esch, T. Depuydt, and D. P. Huyskens, "The use of an aSi-based EPID for routine absolute dosimetric pre-treatment verification of dynamic IMRT fields," Radiother. Oncol. 71, 223-234 (2004).

${ }^{6}$ M. Wendling, R. J. W. Louwe, L. N. McDermott, J. Sonke, M. van Herk, and B. J. Mijnheer, "Accurate two-dimensional IMRT verification using a back-projection EPID dosimetry method," Med. Phys. 33, 259-273 (2006).

${ }^{7}$ Y. El-Mohri, L. E. Antonuk, J. Yorkston, K.-W. Jee, M. Maolinbay, K. L. Lam, and J. H. Siewerdsen, "Relative dosimetry using active matrix flatpanel imager (AMFPD) technology," Med. Phys. 26, 1530-1541 (1999).

${ }^{8}$ J. M. Moran, D. A. Roberts, T. S. Nurushev, L. E. Antonuk, Y. El-Mohri,
} 
and B. A. Fraass, "An Active Matrix Flat Panel Dosimeter (AMFPD) for in-phantom dosimetric measurements," Med. Phys. 32, 466-472 (2005).

${ }^{9}$ L. E. Antonuk, Y. El-Mohri, W. Huang, K. Jee, J. H. Siewerdsen, M. Maolinbay, V. E. Scarpine, H. Sandler, and J. Yorkston, "Initial performance evaluation of an indirect-detection, active matrix flat-panel imager (AMFPI) prototype for megavoltage imaging," Int. J. Radiat. Oncol. Biol. Phys. 42, 437-452 (1998).

${ }^{10}$ L. E. Antonuk, Y. El-Mohri, J. H. Siewerdsen, J. Yorkston, W. Huang, and V. E. Scarpine, "Empirical investigation of the signal performance of a high-resolution, indirect detection, active matrix flat-panel imager (AMFPI) for fluoroscopic and radiographic operation," Med. Phys. 24, 51-70 (1997).

${ }^{11}$ D. A. Low, W. B. Harms, S. Mutic, and J. A. Purdy, "A technique for the quantitative evaluation of dose distributions," Med. Phys. 25, 656-661 (1998).

${ }^{12}$ A. Bakai, M. Alber, and F. Nüsslin, "A revision of the $\gamma$-evaluation con- cept for the comparison of dose distributions," Phys. Med. Biol. 48, 3543-3553 (2003).

${ }^{13}$ J. M. Moran, J. Radawski, and B. A. Fraass, "A dose-gradient analysis tool for IMRT QA,” J. Appl. Clin. Med. Phys. 6, 62-73 (2005).

${ }^{14}$ D. Litzenberg, J. M. Moran, and B. A. Fraass, "Incorporation of realistic delivery limitations into dynamic MLC treatment delivery," Med. Phys. 29, 810-820 (2002).

${ }^{15}$ N. Dogan, L. B. Leybovich, and A. Sethi, "Comparative evaluation of Kodak EDR2 and XV2 films for verification of intensity modulated radiation therapy," Phys. Med. Biol. 47, 4121-4130 (2002).

${ }^{16} \mathrm{~S}$. Steciw, B. Warkentin, S. Rathee, and B. G. Fallone, "Threedimentional IMRT verification with a flat-panel EPID," Med. Phys. 32, 600-612 (2005).

${ }^{17}$ W. Ansbacher, "Three-dimensional portal image-based dose reconstruction in a virtual phantom for rapid evaluation of IMRT plans," Med. Phys. 33, 3369-3382 (2006). 